\title{
IHMPC Aplicado em um Reator de Polimerização de Estireno Sujeito a Falhas Operacionais
}

\author{
Rodrigo Ribeiro Santos* Márcio A. F. Martins* \\ Oscar A. Z. Sotomayor ${ }^{* *}$ \\ * Programa de Pós-Graduação em Mecatrônica, Escola Politécnica, \\ Universidade Federal da Bahia, Salvador, BA \\ (e-mail: rodrigoribeiro@ufba.br) (e-mail:marciomartins@ufba.br). \\ ${ }^{* *}$ Departamento de Engenharia Elétrica, Universidade Federal de \\ Sergipe, São Cristóvão, SE (e-mail: oscars@del.ufs.br).
}

\begin{abstract}
This paper presents the development of a stabilizing model predictive control (IHMPC) based fault diagnosis system with observer of unknown input. The proposed system acts as an operational supervisor in order to monitor process, and actuator and sensor faults. The passive fault tolerance characteristics associated with IHMPC are analyzed for different types of faults in a styrene polymerization reactor. The results obtained by numerical simulation show that for all faults scenarios the fault tolerance based IHMPC maintains plant stability even when the process performance is degraded with sensor faults.

Resumo: Neste trabalho é apresentado o desenvolvimento de um sistema de diagnóstico de falhas com observador de entrada desconhecida associado ao controle preditivo com estabilidade garantida (IHMPC). O sistema proposto atua como supervisor operacional, a fim de monitorar falhas no processo, em atuador e em sensor. As características de tolerância a falhas passivas associadas ao IHMPC são analisadas para diferentes tipos de falhas em um reator de polimerização de estireno. Os resultados obtidos por simulação numérica mostram que, para todos os cenários de falhas, a tolerância a falhas baseada no IHMPC mantém a estabilidade da planta mesmo quando o desempenho do processo é degradado com falhas em sensor.
\end{abstract}

Keywords: Model predictive control; Stability; Fault diagnosis; Unknown input observer; Styrene polymerization reactor.

Palavras-chaves: Controle preditivo; Estabilidade; Diagnóstico de falhas; Observador de entrada desconhecida; Reator de polimerização de estireno.

\section{INTRODUÇÃO}

A necessidade de obter maiores níveis em segurança operacional, melhoria da confiabilidade e elevação dos lucros na indústria de processos depende diretamente de sistemas de controle automatizados. Apesar dos benefícios que a automação fornece, as plantas industriais são susceptíveis a problemas ocasionados por falhas operacionais, envolvendo falhas em sensores, em atuadores ou em componentes internos do próprio processo (Blanke et al., 2016).

Falha é um evento anormal ou um desvio não aceitável de pelo menos uma propriedade característica do sistema (Zhang and Jiang, 2008). Nessa situação, a operação do processo é conduzida a um ponto diferente do desejado, na qual o sistema de controle pode não atuar conforme os requisitos de desempenho. Caso essa situação não seja corrigida por uma ação apropriada, graves consequências emergenciais podem ocorrer, ocasionando perdas econômicas na produção, danos aos equipamentos da planta, violações de regras de segurança, desastres ambientais ou, até mesmo, comprometer vidas humanas. Nessa perspectiva, observa-se na literatura esforços para desenvolver métodos que possibilitem o projeto de sistemas de controle capazes de compensar automaticamente os efeitos de falhas operacionais, enquanto a planta estiver operando em uma região ainda controlável (Yu and Jiang, 2015). Dessa forma, garante-se uma resposta eficiente e uma recuperação do processo em tempo adequado, prevenindo a propagação de falhas incipientes locais para falhas graves globais. Esses sistemas são denominados de Controle Tolerante a Falhas (Fault Tolerant Control - FTC).

Segundo Hartley and Maciejowski (2014), o controle preditivo (Model Predictive Control - MPC), que tem sido amplamente adotado pela indústria de processos como estratégia de controle avançado, fornece uma arquitetura de implementação adequada para o FTC. O MPC, em cada intervalo de amostragem, aplica uma ação de controle que é obtida pela solução de um problema de otimização, minimizando uma função de custo sujeita a restrições. Como apenas a primeira ação de controle da trajetória ótima é aplicada na planta e, no intervalo seguinte, o procedimento é recalculado usando novas medições, então o problema de otimização pode ser modificado devido ao conhecimento sobre falhas na planta. Dessa forma, a redundância física 
e/ou analítica dentro da planta pode ser explorada para recuperar um nível aceitável de desempenho. O sistema FTC pode ser passivo (Passive FTC - PFTC) ou ativo (Active FTC - AFTC), o primeiro compensa as falhas através da robustez do controlador para garantir que a planta mantenha a estabilidade, já o segundo reconfigura o sistema de controle, de modo que além da estabilidade da planta e o desempenho possa ser mantido.

Em particular, o objetivo desse trabalho é implementar uma estrutura PFTC através da estratégia MPC com horizonte de predição infinito (Infinite Horizon MPC IHMPC) para compensar um conjunto de falhas de modo que a estabilidade da planta seja preservada. O sistema de supervisão com método de detecção e diagnóstico de falhas (Fault Detection and Diagnosis - FDD) será baseado em banco de Observadores de Entradas Desconhecidas.

O estudo de caso desse trabalho é relacionado ao controle do reator de polimerização de estireno. As características desse processo apresentam diversos desafios, como não-linearidade, estrutura multivariada, potencial instabilidade, múltiplos estados estacionários e alta sensibilidade quanto a mudança no ponto de operação (McAfee et al., 2016), ocasionado, por exemplo, por falhas operacionais. Essas falhas podem ser relacionadas a alteração de parâmetros do modelo do processo, mudança na dinâmica operacional de atuadores e/ou medição incorreta de sensores.

No trabalho de Sotomayor and Odloak (2005) foi proposta uma metodologia baseada em observador para diagnóstico de falhas em plantas químicas, entretanto para o processo de polimerização de estireno abordou-se somente falhas no processo. Por outro lado, Alvarez and Odloak (2012) propuseram uma estrutura de otimização para o processo de polimerização, incluindo a rejeição de perturbações, no entanto as falhas operacionais não foram levadas em consideração. Por fim, Picó and Adam (2015) analisaram o desempenho de um banco de observadores para diagnóstico de falhas apenas em sensores de um reator tanque agitado contínuo (Continuous Stirred Tank Reactor - CSTR).

Em face do exposto, a principal contribuição desse trabalho é o desenvolvimento de um sistema PFTC, baseado em uma estrutura de controle preditivo com garantia de estabilidade, aplicado ao processo de polimerização de estireno sujeito a falhas no processo, em atuadores e em sensores, com supervisão operacional de um sistema FDD.

\section{SISTEMA DE DIAGNÓSTICO DE FALHAS}

O sistema FDD foi baseado em Observador de Entrada Desconhecida (Unknown Input Observer - UIO), que utiliza o princípio de desacoplamento de perturbação (Chen and Patton, 1999). Assim, um residual é projetado, no caso ideal, desacoplando os efeitos de falhas de entradas desconhecidas (distúrbios, ruídos) ou desacoplando os efeitos das próprias falhas para fins de isolamento.

O observador consiste em um sistema de redundância analítica formado por um modelo matemático paralelo ao processo com realimentação do erro de estimação da saída. O residual $(r)$ gerado é definido para indicar ausência ou presença de falhas. O método mais comum para tomada de decisão é através de uma variável de decisão binária $\left(S_{r}\right)$, sendo usado um limiar $(T)$ na função de avaliação do residual $(J)$. O UIO é uma generalização do observador de Luenberger, que é usado para resolver o problema de geração residual robusta, sendo designado como Observador de Detecção de Falha de Entrada Desconhecida (Unknown Input Fault Detection Observer - UIFDO).

Aqui, o escopo é estender a abordagem proposta por Sotomayor and Odloak (2005) e projetar um sistema FDD geral para estudo de caso no reator de polimerização de estireno. Assim, pretende-se diagnosticar falhas não somente em parâmetros do modelo, mas também em atuador e em sensor. Para o desenvolvimento do UIFDO o modelo do processo é representado conforme (1).

$$
\begin{aligned}
x(k+1) & =A x(k)+B u(k)+E f(k) \\
y(k) & =C x(k)
\end{aligned}
$$

Sendo: $x, u, y$ e $f$, os vetores, respectivamente, de estado, de entrada, de saída e de falha e $A, B, C$ e $E$ matrizes conhecidas de dimensões apropriadas. Para realizar a isolação de falhas, ou seja, determinar uma falha ocorrida em conjunto conhecido, o vetor $f$ é particionado em $\left[\begin{array}{ll}f_{1} & f_{2}\end{array}\right]^{T}$, sendo $f_{1}$ relacionado as falhas que serão insensíveis ao detector e $f_{2}$ o conjunto de falhas que serão monitoradas. Também, a matriz $E$ é particionada em $\left[\begin{array}{ll}E_{1} & E_{2}\end{array}\right]$.

Em diversas aplicações a informação sobre todos os estados do sistema pode não ser necessária ou até mesmo impossível de se obter, como, por exemplo, modelos obtidos por linearização de sistemas não-lineares complexos. Dessa forma, a observação de uma parcela ou combinação linear dos estados é suficiente para geração residual (Chen and Patton, 1999). Com isso, a solução para o problema do observador pode ser obtida sob condições de existência menos restritivas do que as impostas pelo observador de ordem completa. Nesse trabalho, utilizou-se o UIFDO de ordem reduzida baseado na abordagem decomposição de valores singulares (Singular Value Decomposition - SVD).

Para isto, considera-se a matriz de transformação nãosingular $T=U_{1}^{T}$, sendo $U_{1}$ obtida através da SVD de $E_{1}$, isto é, $E_{1}=U_{1}\left[\begin{array}{c}\Sigma_{1} \\ 0\end{array}\right] V_{1}^{T}$. Assim, aplicando a transformação de estado $z=T x$, obtém-se:

$$
\begin{aligned}
z(k+1) & =T A T^{-1} z(k)+T B u(k)+T E_{1} f_{1}(k)+T E_{2} f_{2}(k) \\
y(k) & =C T^{-1} z(k)
\end{aligned}
$$

Definindo as seguintes partições:

$$
\begin{gathered}
z(k)=\left[\begin{array}{l}
z_{1}(k) \\
z_{2}(k)
\end{array}\right] \quad T A T^{-1}=\left[\begin{array}{ll}
A_{11} & A_{12} \\
A_{21} & A_{22}
\end{array}\right] \quad T B=\left[\begin{array}{l}
B_{1} \\
B_{2}
\end{array}\right] \\
C T^{-1}=\left[\begin{array}{ll}
C_{1} & C_{2}
\end{array}\right] \quad T E_{1}=\left[\begin{array}{c}
E_{11} \\
0
\end{array}\right] \quad T E_{2}=\left[\begin{array}{l}
E_{21} \\
E_{22}
\end{array}\right]
\end{gathered}
$$

Então, o sistema transformado pode ser dividido em dois subsistemas. O subsistema $1, z_{1}(k+1)$, é afetado por $f_{1}$ e $f_{2}$, enquanto que o subsistema $2, z_{2}(k+1)$, é afetado apenas por $f_{2}$. Assumindo que os estados do subsistema 1 sejam calculados usando medições de saída, então o subsistema 2 pode ser obtido e um gerador residual baseado em observador pode ser elaborado para ser insensível a $f_{1}$. Se a partição $C_{1}$ possuir posto completo, então $z_{1}(k)$ poderá ser eliminado de $z_{2}(k+1)$ obtendo-o por meio de $y(k)$. 
Caso $C_{1}$ não possua posto completo, deve-se considerar a matriz de transformação não singular $T_{1}=U_{2}^{T}, \operatorname{com} U_{2}$ obtida a partir da SVD de $C_{1}=U_{2}\left[\begin{array}{c}\Sigma_{2} \\ 0\end{array}\right] V_{2}^{T}$. Aplicando a transformação $y^{*}=T_{1} y$ em (2):

$$
y^{*}(k)=U_{2}^{T} y(k)=\left[\begin{array}{c}
\Sigma_{2} \\
0
\end{array}\right] V_{2}^{T} z_{1}(k)+U_{2}^{T} C_{2} z_{2}(k)
$$

Separando os subsistemas de (3):

$$
\begin{aligned}
& y_{1}^{*}(k)=\Sigma_{2} V_{2}^{T} z_{1}(k)+C_{21} z_{2}(k) \\
& y_{2}^{*}(k)=C_{22} z_{2}(k)
\end{aligned}
$$

Agora, substituindo $z_{1}(k)$ de (4) no subsistema 2 em $(2)$ e com uso do observador de Luenberger, define-se o UIFDO:

$$
\begin{aligned}
\hat{z}_{2}(k+1) & =\bar{A}_{22} \hat{z}_{2}(k)+B_{2} u(k)+A_{21}\left(\Sigma_{2} V_{2}^{T}\right)^{+} y_{1}^{*}(k) \\
& +K\left(y_{2}^{*}(k)-\bar{C}_{22} \hat{z}_{2}(k)\right) \\
r(k) & =y_{2}^{*}(k)-\bar{C}_{22} \hat{z}_{2}(k)
\end{aligned}
$$

Sendo: $\bar{A}_{22}=A_{22}-A_{21}\left(\Sigma_{2} V_{2}^{T}\right)^{+} \bar{C}_{21}$ e $\bar{C}_{22}=C_{22}$, com $(+)$ denotando a matriz pseudo-inversa e $K$ o ganho do observador. Maiores detalhes sobre a teoria do observador consultar Sotomayor and Odloak (2005).

O diagnóstico da falha é realizado através do cálculo de $z_{1}(k)$ em (4) e posterior substituição no subsistema 1 de (2), conforme indicado em (8). Como a estimativa da magnitude da falha no instante atual depende da informação de $z_{1}(k+1)$, então o diagnóstico da falha é atrasado em um intervalo de amostragem.

$$
\begin{aligned}
\hat{f}_{1}(k)=\left(E_{11}\right)^{+}\left[\hat{z}_{1}(k+1)\right. & -A_{11} \hat{z}_{1}(k) \\
& \left.\left.-A_{12} \hat{z}_{2}(k)\right)-B_{1} u(k)\right]
\end{aligned}
$$

Para o caso de falhas no processo e no atuador, o modelo definido em (1) pode ser diretamente aplicado. Já para falha em sensor, o modelo foi estendido para:

$$
\begin{aligned}
x(k+1) & =A x(k)+B u(k)+E_{a} f_{a}(k)+E_{p} f_{p}(k) \\
y(k) & =C x(k)+E_{s} f_{s}(k)
\end{aligned}
$$

Sendo: $f_{a}, f_{p}, f_{s}$ falhas relacionadas ao atuador, processo e sensor, respectivamente, e suas respectivas matrizes de distribuição de falhas. De acordo com Noura et al. (2009), falhas em sensores podem ser descritas como:

$$
f_{s}(k+1)=f_{s}(k)+T_{s} \xi(k)
$$

Sendo $\xi(k)$ uma função escalar que representa a evolução da falha no sensor. Assim, combinando (9) e (10):

$$
\begin{aligned}
{\left[\begin{array}{c}
x(k+1) \\
f_{s}(k+1)
\end{array}\right]=} & {\left[\begin{array}{cc}
A & 0 \\
0 & 1
\end{array}\right]\left[\begin{array}{l}
x(k) \\
f_{s}(k)
\end{array}\right]+\left[\begin{array}{c}
B \\
0
\end{array}\right] u(k)+\left[\begin{array}{c}
E_{a} \\
0
\end{array}\right] f_{a}(k) } \\
& +\left[\begin{array}{c}
E_{p} \\
0
\end{array}\right] f_{p}(k)+\left[\begin{array}{c}
0 \\
T_{s}
\end{array}\right] \xi(k) \\
y(k)= & {\left[C E_{s}\right]\left[\begin{array}{l}
x(k) \\
f_{s}(k)
\end{array}\right] }
\end{aligned}
$$

Como o modelo em (11) possui a mesma estrutura de espaço de estados de (1), então o procedimento de desacoplamento de falha descrito anteriormente poderá ser aplicado. Porém, a estimativa da magnitude da falha do sensor é realizada com a transformação inversa do estado $z(k)$ para $x(k)$, pois neste caso a falha do sensor pertence ao vetor de estado do sistema.

Para o sistema FDD aplicado nesse trabalho, implementouse um banco com três UIFDO para produzir um conjunto residual estruturado, sendo cada observador projetado para ser insensível a uma falha específica e sensível as demais falhas. Desta forma, é possível isolar unicamente uma única falha em determinado momento. Assumiu-se insignificante a probabilidade de falhas simultâneas.

\section{SISTEMA DE CONTROLE}

Os requisitos de qualidade da produção do polímero de estireno estão diretamente relacionados à operação funcional do reator de polimerização. O objetivo do sistema de controle é buscar a menor variabilidade possível no ponto operacional desejado, manter a estabilidade da planta e atender as restrições operacionais.

Nesse trabalho foi implementado um sistema de controle multivariável com duas entradas e duas saídas, baseado no algoritmo do IHMPC nominalmente estável proposto por Odloak (2004). O projeto do controlador incorpora o modelo do processo, descrito no trabalho de Sotomayor and Odloak (2005), como apresentado na Tabela 1. Embora o modelo dinâmico do reator esteja na forma de função de transferência, o IHMPC considerado aqui é baseado no modelo de espaço de estados sintetizado a partir da expressão analítica de resposta ao degrau de sistemas com polos distintos e estáveis em malha aberta.

Tabela 1. Modelo de predição do IHMPC.

\begin{tabular}{ccc}
\hline & $u_{1}\left(Q_{i}\right)[\mathrm{L} / \mathrm{h}]$ & $u_{2}\left(Q_{c}\right)[\mathrm{L} / \mathrm{h}]$ \\
\hline$y_{1}(\eta)[\mathrm{L} / \mathrm{g}]$ & $\frac{-45,37}{5,795 s+1} e^{-1,547 s}$ & $\frac{3,66}{9,098 s+1} e^{-4,169 s}$ \\
$y_{2}\left(T_{e}\right)[\mathrm{K}]$ & $\frac{121,17}{7,049 s+1} e^{-0,771 s}$ & $\frac{-38,88}{7,206 s+1} e^{-1,768 s}$ \\
\hline
\end{tabular}

O IHMPC é baseado na solução, em cada intervalo de amostragem, do problema de otimização (12).

$$
\begin{array}{r}
\min _{\Delta u_{k}, \delta_{y, k}} \sum_{j=0}^{m}\left\|e(k+j \mid k)-\delta_{y, k}\right\|_{Q}^{2}+\left\|x^{d}(k+m \mid k)\right\|_{\bar{Q}}^{2} \\
+\sum_{j=0}^{m-1}\|\Delta u(k+j \mid k)\|_{R}^{2}+\left\|\delta_{y, k}\right\|_{S}^{2}
\end{array}
$$

Sujeito a:

$$
\left\{\begin{array}{l}
-\Delta u_{\max } \leq \Delta u(k+j \mid k) \leq \Delta u_{\max } \quad j=0, \ldots, m-1 \\
u_{\min } \leq \Delta u(k-1)+\sum_{i=0}^{j} \Delta u(k+i \mid k) \leq u_{\max } \\
\Delta u(k+j \mid k)=0 \quad j \geq m \\
x^{s}(k+m \mid k)-y_{s p}-\delta_{y, k}=0
\end{array}\right.
$$

Sendo: $e(k+j \mid k)=y(k+j \mid k)-y_{s p}$ o erro de predição de saída, $y_{s p}$ a referência da saída, $m$ o horizonte de controle, $\underline{Q}, R$ e $S$ matrizes de ponderação positivas definidas, $\bar{Q}$ matriz de peso do custo terminal, obtida pela equação de Lyapunov do sistema, $x^{s}$ corresponde aos estados integradores introduzidos pela forma incremental da entrada, $x^{d}$ corresponde aos estados estáveis, $\delta_{y, k}$ é o vetor de variáveis de folga (slacks) e $k$ é o intervalo 
de tempo discreto. As variáveis de folga permitem que o controlador seja aplicado aos casos em que não há graus de liberdade suficientes para zerar o erro no estado estacionário em todas as saídas do sistema.

O problema de otimização (12), sujeito as restrições (13), é resolvido por programação quadrática. O primeiro elemento da sequência do sinal de controle obtido é aplicado à planta. O IHMPC desenvolvido usando predições baseadas no modelo de espaço de estado incremental, obtido através da conversão das funções de transferência, fornece ação integral que elimina o erro de estado estacionário. Os estados do modelo são atualizados a cada intervalo de amostragem usando Filtro de Kalman. Maiores detalhes sobre a teoria do IHMPC podem ser consultados em Odloak (2004). A sintonia do IHMPC foi realizada através de testes de simulação numérica, sendo selecionados: intervalo de amostragem $T_{s}=1 \mathrm{~h}$, horizonte de controle $m=3$, matrizes de ponderação $Q=\left[\begin{array}{ll}8 & 1\end{array}\right], R=\left[\begin{array}{ll}800 & 800\end{array}\right]$ e $S=\left[\begin{array}{ll}10^{4} & 10^{4}\end{array}\right]$.

\section{REATOR DE POLIMERIZAÇÃO}

A operação funcional do reator de polimerização de estireno é complexa, pois envolve reações exotérmicas, cinética de reação desconhecida e alta viscosidade (Maner et al., 1996). Além disso, caso o processo não esteja operando dentro das especificações desejadas, pode ser difícil determinar qual fator é responsável pela anormalidade. Ademais, segundo McAfee et al. (2016) a impossibilidade de purificar produtos que estão fora de especificação faz com que um sistema de supervisão da operação seja recomendado. O modelo fenomenológico não-linear do reator de polimerização utilizado nesse trabalho é o descrito por Maner et al. (1996). Detalhes sobre o modelo, equações e valores dos parâmetros consultar a referência citada.

O reator possui três entradas de alimentação: monômero de estireno puro, iniciador dissolvido em benzeno e solvente de benzeno. O processo é realizado em um procedimento de três etapas: iniciação, propagação e término. O calor excedente gerado pelas reações exotérmicas é removido pelo fluido de arrefecimento na camisa do reator. As variáveis controladas do processo são: viscosidade intrínseca $(\eta)$ e temperatura do reator $\left(T_{e}\right)$. Para isto, o sistema de controle manipula a vazão do iniciador $\left(Q_{i}\right)$ e a vazão do fluido de arrefecimento $\left(Q_{c}\right)$. Na operação do reator foram consideradas falhas no processo (fator de frequência $-A_{t}$ ), no atuador (válvula de vazão do iniciador $Q_{i}$ ) e no sensor (saída de viscosidade intrínseca $\eta$ ), formando assim o vetor de falhas $f=\left[\begin{array}{lll}A_{t} & Q_{i} & \eta\end{array}\right]$. O modelo descrito por Maner et al. (1996) foi linearizado na forma de (1). A arquitetura do sistema PFTC é apresentada na Figura 1. O sistema de diagnóstico de falhas possui a função de supervisão operacional, para fornecer informações referentes a estimativas de falhas na planta.

Devido às características não-lineares do reator, para reduzir o desequilíbrio entre planta e modelo, nos observadores foi utilizado um ganho adaptativo $(K)$ na forma do filtro do Kalman, conforme apresentado em (14) e (15), sendo $P$ a matriz de covariância do erro de predição.

$$
\begin{aligned}
K(k) & =\left(\bar{A}_{22} P(k-1) C_{22}^{T}\right)\left(I+C_{22} P(k-1) C_{22}^{T}\right)^{-1} \\
P(k) & =\left(\bar{A}_{22}-K(k) C_{22}\right) P(k-1) \bar{A}_{22}^{T}
\end{aligned}
$$

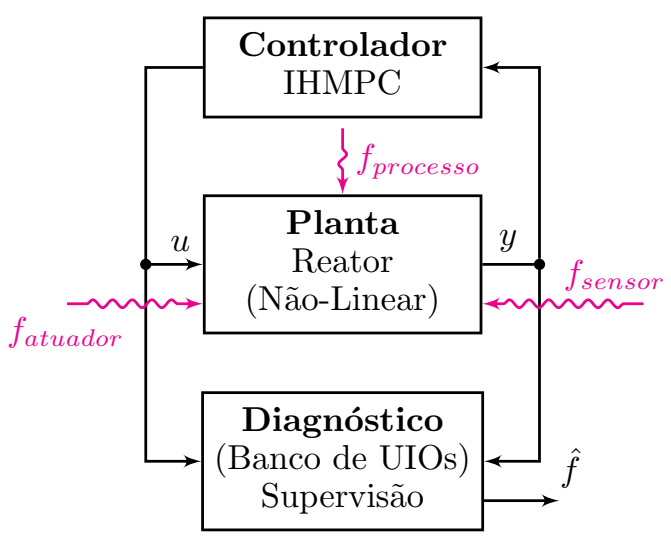

Figura 1. Estrutura PFTC com supervisão operacional.

\section{RESULTADOS E DISCUSSÃO}

\subsection{Análise de falha no parâmetro do processo $A_{t}$}

A modelagem do processo de polimerização de estireno possui considerável incerteza, principalmente nas constantes de velocidade de propagação e terminação. Sendo assim, esta análise de falha considera uma mudança abrupta na constante de taxa de terminação, através da variação do fator de frequência na equação para reação terminal $\left(A_{t}\right)$. Segundo Xu et al. (2017), devido à cinética do processo, o valor desse parâmetro pode sofrer redução por causa das limitações de difusão em conversões de monômeros.

Nesse cenário foi considerada uma redução abrupta de $5 \%$ no parâmetro $A_{t}$ do modelo do processo, ocorrendo no instante de tempo $40 h$ na simulação numérica. $\mathrm{Na}$ Figura 2, verifica-se que o sistema de controle acomodou a falha na operação do reator. As variáveis de saída $\eta$ e $T_{e}$ aumentaram como consequência da anormalidade e para corrigir essa situação o controlador aumentou os sinais das variáveis manipuladas. O tempo de acomodação do processo foi em torno de 18 instantes de amostragem.
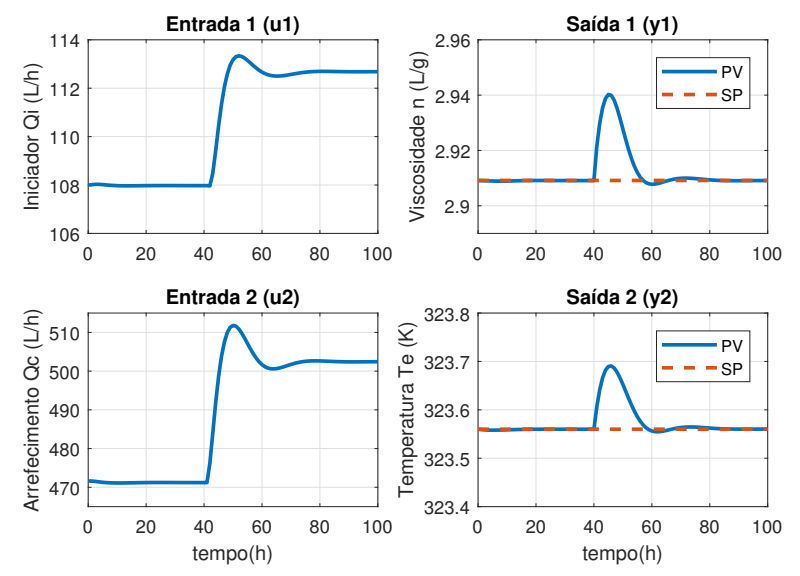

Figura 2. Ação do IHMPC para falha no processo (- $5 \%$ de $A_{t}$ ) ocorrida no instante $40 \mathrm{~h}$.

Como verificado na Figura 3, a falha no processo foi isolada dentro do conjunto possível, pois foi gerado um alarme dos residuais do atuador e do sensor, $r_{Q_{i}}$ e $r_{\eta}$, e não pelo residual do processo $r_{A_{t}}$, devido a característica de insensibilidade a falha do detector. Usando um limite 
de decisão $T=0,1$, determinado experimentalmente, a detecção e o isolamento dessa falha foram realizados conforme objetivo almejado. Ainda na Figura 3 constatase que a estimativa da falha foi obtida em torno de 10 instantes de amostragem, ou seja, informação antecipada antes da acomodação das saídas, de forma a conhecer as causas da variação nas controladas.
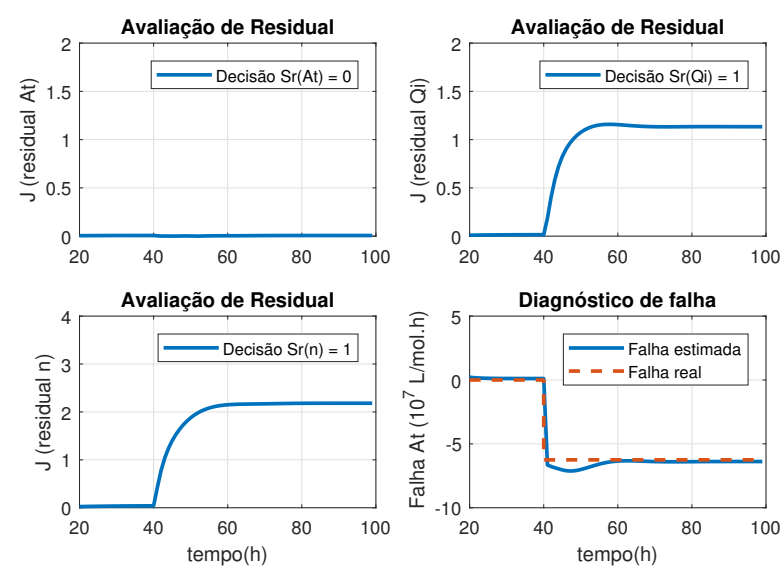

Figura 3. Diagnóstico para falha no processo (- $5 \%$ de $A_{t}$ ).

\subsection{Análise de falha no atuador da entrada $Q_{i}$}

Falha em atuador conduz a planta para desempenho fora das especificações desejadas e ainda a torna parcialmente incontrolável. No reator de polimerização, falha na dinâmica do atuador que manipula a entrada $u_{1}$ provoca alteração na vazão de entrada do iniciador $\left(Q_{i}\right)$. Nesse cenário de falha, com uma possível redução na taxa de variação do atuador (válvula de controle), ocorre diminuição da concentração de radicais e a taxa de liberação de calor pela reação diminui, reduzindo assim a temperatura do reator e aumentando a viscosidade do polímero produzido.

No presente caso, o ganho do atuador de $Q_{i}$ foi reduzido em $10 \%$, ocorrendo de forma abrupta a partir do instante $40 h$ na simulação. Verifica-se na Figura 4 que o IHMPC compensou os efeitos da falha aumentando o esforço de controle na própria entrada $u_{1}$, ou seja, com uma dinâmica $10 \%$ abaixo do normal, o controlador aumentou a ação de controle para compensar a perda parcial de efetividade na atuação da válvula de controle. O sistema de controle também atuou na entrada $u_{2}$, de forma a manter a saída $T_{e}$ em seu ponto de ajuste. Verifica-se nesse cenário que o IHMPC exibiu um grau intrínseco de tolerância a falhas do atuador, mesmo quando a falha não era conhecida pelo controlador. Por fim, nota-se que para atingir o estado estacionário foram necessários 35 instantes de amostragem e o desempenho dinâmico, após a estabilização da planta, não foi degradado.

$\mathrm{Na}$ Figura 5 é apresentado que os residuais $r_{A_{t}}$ e $r_{\eta}$ foram ativados enquanto $r_{Q_{i}}$ permaneceu inativo, o que caracteriza a assinatura da falha do atuador. A detecção e o diagnóstico desta falha foram realizados em torno de 17 instantes de amostragem. O erro reduzido na estimação de magnitude da falha é explicado devido às incertezas do modelo de aproximação linear usado pelo observador em relação ao modelo não-linear da planta.
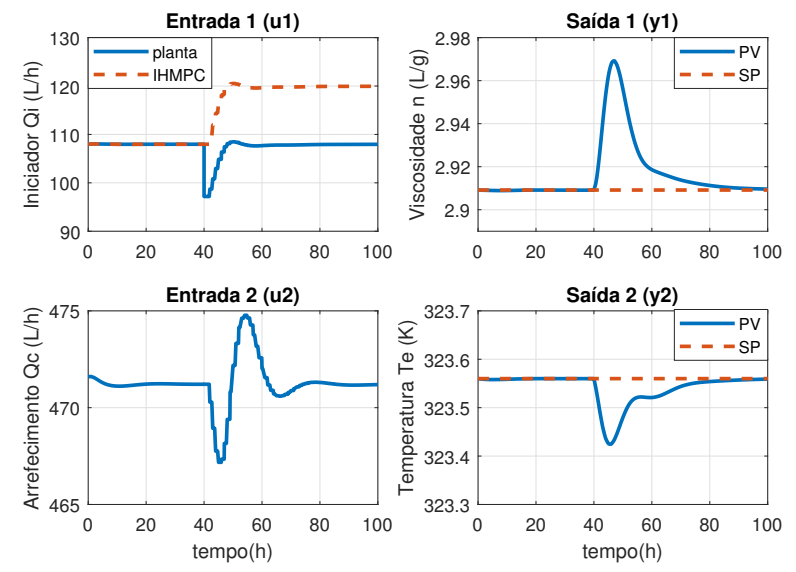

Figura 4. Ação do IHMPC para falha no atuador $(-10 \%$ de $Q_{i}$ ) ocorrida no instante $40 \mathrm{~h}$.
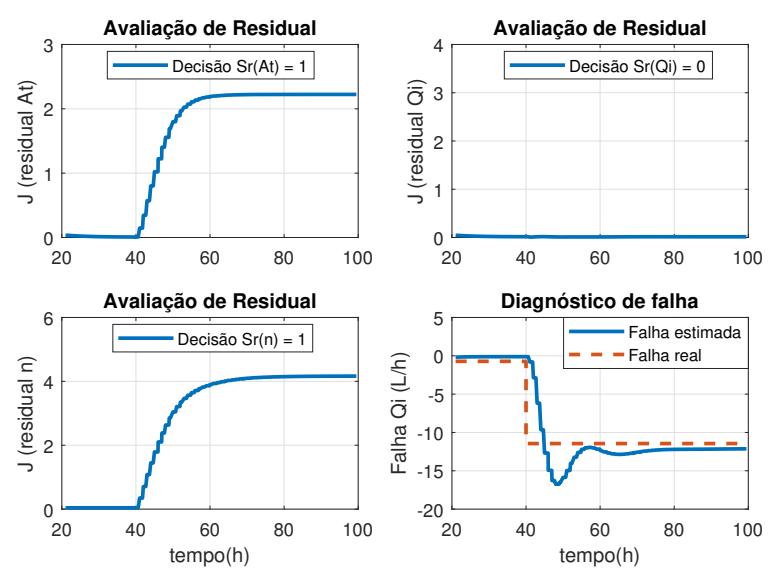

Figura 5. Diagnóstico da falha no atuador $\left(-10 \%\right.$ de $\left.Q_{i}\right)$.

\subsection{Análise de falha no sensor da saída $\eta$}

A medição de variáveis em reatores de polimerização é uma tarefa desafiadora devido à complexidade dos mecanismos físicos e da cinética do processo. Segundo Richards and Congalidis (2006), nesses reatores diversas variáveis importantes não podem ser medidas on-line, tal como o peso molecular médio, ou são medidas com susceptibilidade a falhas, como a viscosidade intrínseca. Em geral, a medição incorreta devido ao funcionamento defeituoso dos sensores conduz a planta para um ponto de operação diferente do ideal, tornando-a ainda parcialmente inobservável. Nessa perspectiva, a situação faltosa simula um viés positivo na medição da viscosidade intrínseca, que pode ser causada, por exemplo, por incrustação de polímero no sensor. A falha foi inserida a partir do instante $40 \mathrm{~h}$, com um aumento abrupto de $5 \%$ no valor da medição da viscosidade.

Através dos resultados apresentados na Figura 6, verificase que mesmo com a ocorrência da falha no sensor da saída $y_{1}$, o controlador manteve a estabilidade da planta. Inicialmente, o IHMPC recebeu a informação da viscosidade com o acréscimo devido a falha no sensor $(P V$ planta com falha). Em seguida, o controlador atuou nas variáveis manipuladas para compensar o desvio de $y_{1} \mathrm{com}$ seu respectivo setpoint $(S P)$, entretanto como o controlador não possui informação sobre a falha, então a medição 
recebida é considerada como verdadeira, assim o modelo teórico do processo é mantido em um viés inferior de $5 \%$ ( $P V$-modelo teórico). Desta forma, o controlador mantém a planta estável, no entanto com o valor real da viscosidade em um ponto diferente do desejado. Nota-se ainda que para acomodar os efeitos da falha na operação do processo, o IHMPC aumentou as ações de controle buscando manter a temperatura no ponto de ajuste. O desvio sistemático de $y_{1}$ é naturalmente explicado pela informação divergente recebida pelo controlador. O grau de tolerância a falhas do IHMPC se mostrou suficiente para estabilizar a planta com tal situação faltosa, porém com prejuízo ao desempenho operacional. Nesse caso, uma estrutura ativa é recomendada, para que o IHMPC possa utilizar a estimativa da falha e realizar procedimentos de reconfiguração, para encontrar uma ação de controle adequada para atingir melhor desempenho operacional.
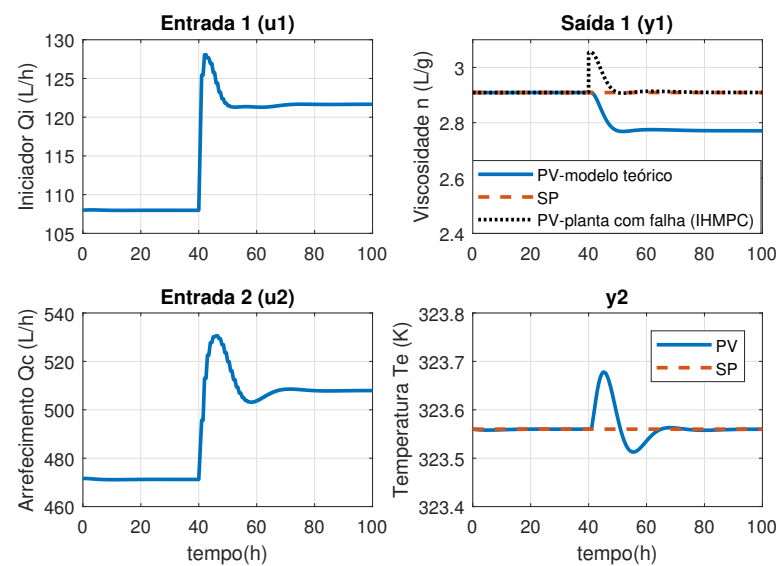

Figura 6. Ação do IHMPC para falha no sensor $(+5 \%$ de $\eta$ ) ocorrida no instante $40 \mathrm{~h}$.
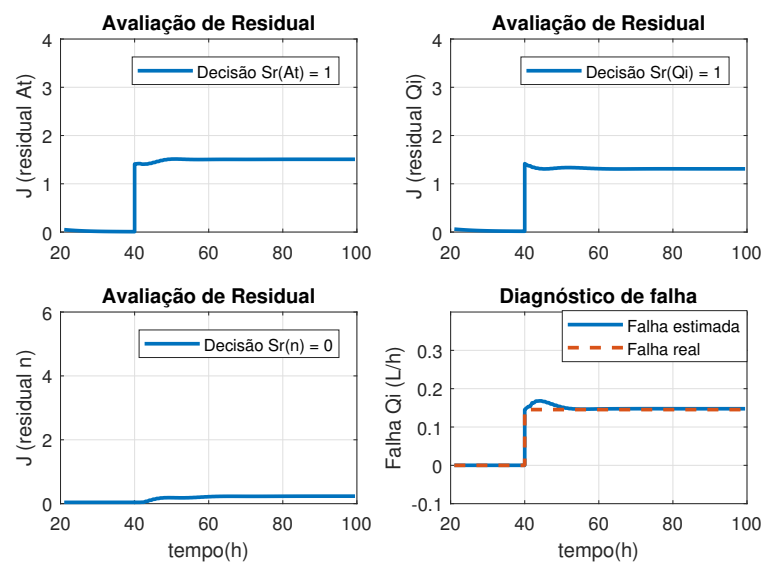

Figura 7. Diagnóstico da falha no sensor $(+5 \%$ de $\eta)$.

A falha no sensor foi detectada e isolada corretamente, conforme apresentado na Figura 7. A magnitude da falha foi estimada em torno de 5 instantes de amostragem. Os resultados fornecidos pelo FDD apresentam as causas para o desempenho operacional degradado do sistema de controle.

\section{CONCLUSÃO}

Neste trabalho foi aplicado o controlador preditivo com estabilidade nominal garantida no processo não-linear de polimerização de estireno associado a sistema de diagnóstico de falhas geral com base em observadores de entrada desconhecidas. Através dos resultados de simulações numéricas foi verificado que para todas as situações de falhas, o sistema de controle conseguiu manter a estabilidade da planta, no entanto para falha em sensor houve degradação de desempenho operacional. Em todos os casos de estudo, o sistema FDD realizou detecção e isolamento em tempo inferior a estabilização da resposta planta e as estimativas da magnitude das falhas foram coerentes com as falhas reais. Como perspectiva futura, sugere-se o desenvolvimento de um FTC ativo com o IHMPC.

\section{REFERÊNCIAS}

Alvarez, L.A. and Odloak, D. (2012). Optimization and control of a continuous polymerization reactor. Brazilian Journal of Chemical Engineering, 29, 807--820.

Blanke, M., Kinnaert, M., Lunze, J., and Staroswiecki, M. (2016). Diagnosis and Fault-Tolerant Control. SpringerVerlag, Berlin.

Chen, J. and Patton, R. (1999). Robust model-based fault diagnosis for dynamic systems. Kluwer Academic, Massachusetts.

Hartley, E. and Maciejowski, J. (2014). Reconfigurable predictive control for redundantly actuated systems with parameterised input constraints. Systems and Control L., 66, 8-15.

Maner, B., Doyle, F., Ogunnaike, B., and Pearson, R. (1996). Nonlinear model predictive control of a simulated multivariable polymerization reactor using secondorder volterra models. Automatica, 32, 1285-1301.

McAfee, T., Leonardi, N., Montgomery, R., Siqueira, J., Zekoski, T., Drenski, F., and Reed, W. (2016). Automatic control of polymer molecular weight during synthesis. Macromolecules, 49, 7170-7183.

Noura, H., Theilliol, D., Ponsart, J., and Chamseddine, A. (2009). Fault-tolerant Control Systems: Design and Practical App. Springer-Verlag, London.

Odloak, D. (2004). Extended robust model predictive control. AIChE J., 50, 1824-1836.

Picó, M.F. and Adam, E.J. (2015). Ftc with observers bank in a cstr. XVI Workshop on Information Processing and Control.

Richards, J.R. and Congalidis, J.P. (2006). Measurement and control of polymerization reactors. Computers and Chemical Eng., 30, 1447-1463.

Sotomayor, O.A.Z. and Odloak, D. (2005). Observer-based fault diagnosis in chemical plants. Chemical Engineering Journal, 112, 93-108.

Xu, C., Wang, J., Gu, X., and Lian, F. (2017). Cfd modeling of styrene polymerization in a cstr. Chemical Engineering Research and Design, 125, 46-56.

$\mathrm{Yu}, \mathrm{X}$. and Jiang, J. (2015). A survey of fault-tolerant controllers based on safety-related issues. Annual Reviews in Control, 39, 46-57.

Zhang, Y. and Jiang, J. (2008). Bibliographical review on reconfigurable fault-tolerant control systems. Annual Reviews in Control, 32, 229-252. 